05

\title{
Микроволновый отклик на переключение намагниченности спиновых вентилей CoFeB/Ta/CoFeB и пленок CoFeB
}

\author{
() А.Д. Таланцев ${ }^{1,2}$, О.В. Коплак' ${ }^{1}$ Г.Л. Львова ${ }^{1,2}$, O.C. Дмитриев ${ }^{2}$, S. Petit Watelot ${ }^{3}$, \\ Y. Lu ${ }^{3}$, S. Mangin ${ }^{3}$, Р.Б. Моргунов ${ }^{1,2,}$ \\ ${ }^{1}$ Институт проблем химической физики РАН, \\ Черноголовка, Россия \\ ${ }^{2}$ Тамбовский государственный технический университет, \\ Тамбов, Россия \\ ${ }^{3}$ Institut Jean Lamour, UMR 7198 CNRS, Université de Lorraine, \\ Nancy, France \\ ฯ E-mail: morgunov2005@yandex.ru
}

(Поступила в Редакцию 16 марта 2017 г.)

$\mathrm{B}$ пленке $\mathrm{MgO} / \mathrm{CoFeB} / \mathrm{MgO} / \mathrm{Ta}$ с одним ферромагнитным слоем и спиновом вентиле $\mathrm{MgO} / \mathrm{CoFeB} / \mathrm{Ta} / \mathrm{CoFeB} /$ $\mathrm{MgO} / \mathrm{Ta}$, состоящем из двух ферромагнитных слоев $\mathrm{CoFeB}$ с монокристаллической структурой, разделенных немагнитным слоем Та, обнаружено отрицательное магнитосопротивление, изменяющее добротность микроволнового резонатора при переключении намагниченности ферромагнитных слоев. Зависимости первой производной поглощения микроволнового поля $\mathrm{dP} / \mathrm{dH}$ от величины постоянного магнитного поля спектрометра в точности воспроизводят петли магнитного гистерезиса образца. Наклон этих зависимостей и амплитуда скачков $\mathrm{dP} / \mathrm{dH}$ при перемагничивании слоев определяются соотношением вкладов отрицательного магнитосопротивления отдельных слоев и положительного гигантского магнитосопротивления многослойной структуры в целом. Обнаруженное явление позволяет использовать поглощение микроволн для создания высокочувствительного бесконтактного индикатора основных состояний намагниченности спинового вентиля.

Работа поддержана Министерством образования и науки РФ (грант 3.1992.2017/ПЧ в рамках конкурса научных проектов, выполняемых научными коллективами исследовательских центров и (или) научных лабораторий образовательных организаций высшего образования.

DOI: 10.21883/FTT.2017.10.44960.082

\section{1. Введение}

Магнитные многослойные гетероструктуры на основе $\mathrm{CoFeB}$ обладают рекордно высокими значениями гигантского магнитосопротивления (ГМС) (до 600\%) [1], что может быть использовано в инженерии разнообразных спиновых логических устройств. Несмотря на то что что структуры на основе $\mathrm{CoFeB}$ изучаются уже давно, интерес к исследованию таких структур только усиливается. Это обусловлено хорошей воспроизводимостью электрических и магнитных свойств гетероструктур при заданных параметрах их роста, которые легко варьировать с целью изменения технических характеристик. Так, например, знак обменного взаимодействия между слоями может быть как положительным, так и отрицательным в зависимости от толщины немагнитной прослойки. Варьирование толщины магнитных слоев $\mathrm{CoFeB}$ позволяет задавать желаемую величину намагниченности насыщения и значения констант анизотропии. Все это позволяет создавать как самые простые структуры с ГМС и осью легкого намагничивания вдоль плоскости, так и совсем нетривиальные образцы, в которых проявляется перпендикулярная анизотропия [2] или взаимодействие Дзялошинского-Мории [3].

Одной из основных характеристик получаемых структур является величина относительного изменения сопро- тивления при переориентации векторов намагниченности ферромагнитных слоев. Широко распространенное измерение сопротивления контактным методом является довольно трудоемкой процедурой и, как правило, требует напыления потенциальных и токовых контактов. Это усложняет технологию датчиков на основе ГМС, препятствует реализации устройств удаленного доступа, требует виртуозной микросистемной инженерии. Как показано далее, о сопротивлении многослойной структуры и ориентации намагниченностей ее ферромагнитных слоев можно судить по микроволновому поглощению, измеряемому в нашей работе в резонаторе спектрометра электронного парамагнитного резонанса. Отклик микроволнового магнитосопротивления структуры на перемагничивание слоев оказывается более сильным, чем соответствующий отклик намагниченности. Поэтому при использовании открытого микроволнового контура, эти результаты дают возможность дистанционного определения магнитного и логического состояния сенсора ГМС, что важно для биологических приложений, широко развиваемых в настоящее время и требующих бесконтактного мониторинга состояний сенсора [4].

Простейшим примером структур с ГМС является структура с двумя ферромагнитными слоями, антиферромагнитно взаимодействующими между собой (рис. 1). 


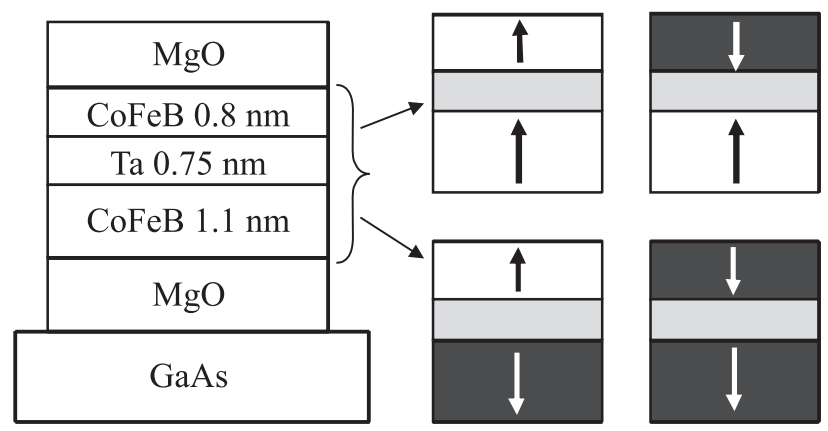

Рис. 1. Схема расположения слоев бислоя (образца I) и направления их намагниченностей в четырех возможных состояниях.

В отсутствие магнитного поля намагниченности слоев такой структуры ориентированы друг против друга, и туннельное сопротивление между слоями максимально. Приложение магнитного поля приводит к перемагничиванию одного из слоев и резкому уменьшению межслоевого сопротивления структуры. Если намагниченности слоев разные, то помимо двух состояний с различной взаимной ориентацией их намагниченностей различают еще два состояния, отвечающие различной ориентации намагниченностей слоев относительно внешнего магнитного поля. Все четыре стабильных состояния гетероструктуры с ГМС схематично показаны на рис. 1. Цель настоящей работы состоит в обнаружении отклика микроволнового поглощения на переключение намагниченности между указанными состояниями.

\section{2. Методика}

Многослойные структуры $\mathrm{MgO}(2.5 \mathrm{~nm}) / \mathrm{CoFeB}(1.1 \mathrm{~nm}) /$ $\mathrm{Ta}(0.75 \mathrm{~nm}) / \mathrm{CoFeB}(0.8 \mathrm{~nm}) / \mathrm{MgO}(2.5 \mathrm{~nm}) / \mathrm{Ta}(5 \mathrm{~nm})$ (образец I - бислой, состоящий их двух ферромагнитных слоев $\mathrm{CoFeB}$, разделенных антиферромагнитным слоем Та) и $\mathrm{MgO}(2.5 \mathrm{~nm}) / \mathrm{CoFeB}(1.1 \mathrm{~nm}) /$ $\mathrm{MgO}(2.5 \mathrm{~nm}) / \mathrm{Ta}(5 \mathrm{~nm})$ (образец II - монослой с одним слоем $\mathrm{CoFeB}$ ) были выращены нами на подложках нелегированного $\mathrm{GaAs}(001)$ методом магнетронного распыления. Подробно методика роста описана в работах $[5,6]$. Гибридизация $3 d$-орбиталей переходных металлов $(\mathrm{Co}, \mathrm{Fe})$ с $\mathrm{O}_{2 p}$-орбиталями $\mathrm{MgO}$ обеспечивает перпендикулярную магнитную анизотропию (ПМА) на границе $\mathrm{CoFeB} / \mathrm{MgO}$ [7]. При малых толщинах слоев $\mathrm{CoFeB}$ (менее $2 \mathrm{~nm}$ ) вклад данного типа анизотропии становится доминирующим, и ось легкого намагничивания направлена перпендикулярно плоскости слоев. Для увеличения ПМА производился быстрый температурный отжиг при $250^{\circ} \mathrm{C}$ в течение $3 \mathrm{~min}$, который приводил к увеличению кристалличности слоев CoFeB. Во время этого процесса диффундирующий бор поглощался слоем Та толщиной $0.75 \mathrm{~nm}$, который также играл роль прослойки между слоями $\mathrm{CoFeB}$, обеспе- чивающей антиферромагнитное взаимодействие между ними $[5,8]$. Характерный размер образцов составлял $0.4 \times 2 \times 2 \mathrm{~mm}$. Петли магнитного гистерезиса были получены с помощью коммерческого СКВИД-магнитометра MPMS 5XL Quantum Design. Микроволновое магнитосопротивление было исследовано с помощью ЭПР-спектрометра $X$-диапазона Bruker ESP 300. Частота микроволнового поля составляла $f_{0}=9447 \mathrm{~Hz}$, мощность $6.3 \mathrm{~mW}$, частота модулирующего поля $100 \mathrm{kHz}$, амплитуда модулирующего поля 10 Ое. Направления электрической и магнитной компонент микроволнового поля относительно направления постоянного поля $H$ спектрометра схематически показаны на рис. 2.

В зависимости от соотношения сопротивлений образца и нагрузки, а также от величины магнитного поля различается три режима, для которых производная микроволновой мощности $\mathrm{dP} / \mathrm{dH}$, измеряемая ЭПР спектрометром, может быть пропорциональна:

1) первой производной магнитной АС-восприимчивости $d \chi_{\mathrm{AC}} / d H$;

2) первой производной сопротивления образца $d \rho / d H$

$3)$ первой производной проводимости образца $d \sigma / d H$.

Первый случай возникает в слабопроводящих или диэлектрических образцах, где вклад в сигнал вносят спины, а роль вихревых токов и магнитосопротивления мала. Этот случай не реализуется в наших экспериментах с металлическими пленками. Второй и третий случаи, как показано в [9], реализуются в проводящих средах (металлах и полупроводниках) в результате изменения добротности резонатора, возникающего вслед-

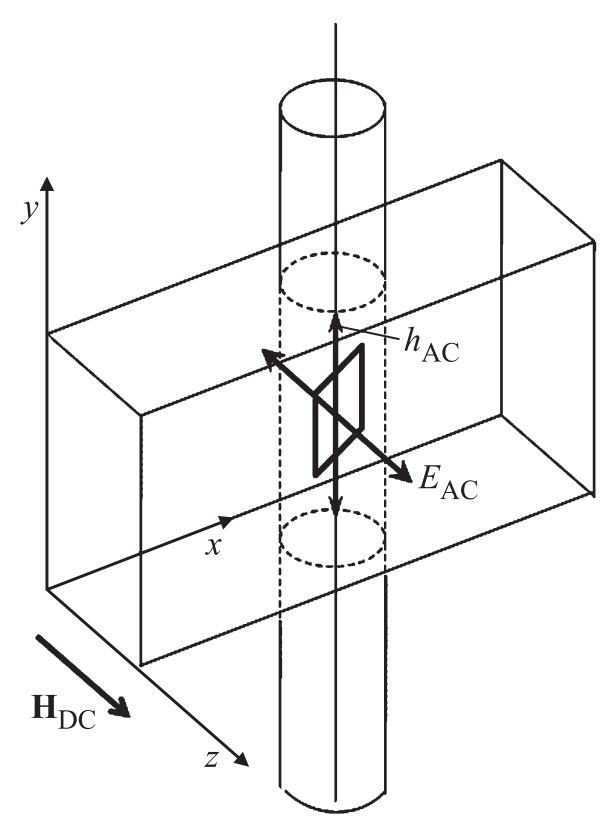

Рис. 2. Схема расположения образца в резонаторе $H_{102}$ при измерениях поглощения микроволнового поля. Магнитная компонента микроволнового поля осциллирует в плоскости $x y$, электрическая - вдоль оси $z$. 

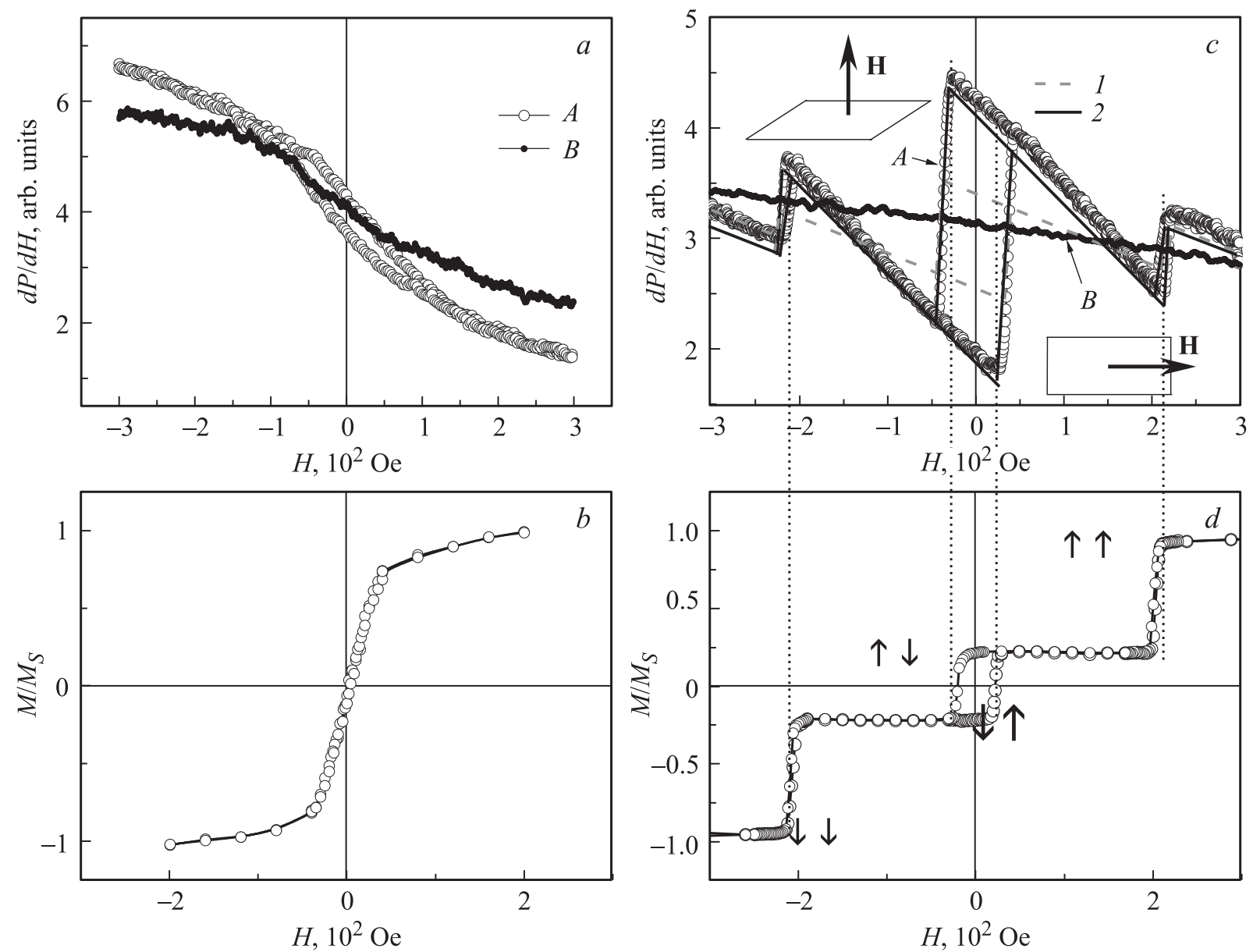

Рис. 3. Зависимости первой производной поглощения микроволнового поля $d P / d H(H)$ для монослоя $(a)$ и бислоя $(c)$ от величины постоянного поля $H$ спектрометра для прямой и обратной разверток магнитного поля при температуре $T=300 \mathrm{~K}$ в двух различных ориентациях магнитного поля относительно плоскости образца: $A-0^{\circ}, B-90^{\circ}$. Линией 1 на части $c$ показана модельная петля гистерезиса $d P / d H(H)$ без учета вклада $Г$ МС, линией 2 - модельная петля гистерезиса $d P / d H(H)$ с учетом ГМС. $(b, d)$ - петли магнитного гистерезиса при температуре $T=300 \mathrm{~K}$ для монослоя и бислоя соответственно. Пунктирными линиями на частях $(c, d)$ показано соответствие между критическими магнитными полями переходов на петле гистерезиса магнитного момента и перепадов на зависимости $d P / d H(H)$.

ствие изменения сопротивления образца в магнитном поле. В работах $[9,10]$ было показано, что для образцов с низким сопротивлением $\rho$ (значительно меньшим сопротивления микроволнового тракта спектрометpa $190 \Omega$ ) поглощаемая мощность $P$ линейно зависит от сопротивления образца и является функцией DC магнитного поля $H: P \sim \rho(H)$. Таким образом, в наших экспериментах производная поглощаемой мощности, измеряемая при наличии модуляции, была прямо пропорциональна производной от сопротивления образца $d P / d H \sim d(\rho(H)) / d H$.

\section{3. Экспериментальные результаты}

Нерезонансное поглощение микроволнового поля было исследовано при $T=300 \mathrm{~K}$ в диапазоне DC-полей от -1000 до +1000 Ое в прямой (слева направо) и обратной (справа налево) развертках магнитного по- ля (рис. 3,a,c). Зависимости первой производной поглощения микроволнового поля от величины DC-поля спектрометра для прямой и обратной разверток магнитного поля были существенно нелинейными как для монослоя, так и для бислоя (рис. $3, a, c$ ). На фоне общей тенденции возрастания $d P / d H$ с уменьшением магнитного поля (развертка справа налево) зависимость $d P / d H$ для монослоя содержит перегибы в положительном и отрицательном полях, близких по величине к полю насыщения намагниченности монослоя (рис. $3, b$ ). Зависимость $d P / d H(H)$ для бислоя (рис. $3, c$ ) содержит три резких скачка в магнитных полях $+200,-25$ и -230 Ое, близких к полям переходов между магнитными состояниями гетероструктуры (петля гистерезиса на рис. $3, d)$. Амплитуды этих скачков $d P / d H$ в полях +200 и -230 Ое, соответствующих полям переходов с перемагничиванием одного ферромагнитного слоя, были примерно вдвое меньше амплитуды скачка $d P / d H$ в 
поле $-25 \mathrm{Oе}$, отвечающего переходу с одновременным перемагничиванием обоих ферромагнитных слоев.

Можно также заметить, что наклон зависимости $d P / d H(H)$ между переходами в полях 200 и в -25 Ое, а также между переходами в полях -25 и -230 Ое (в этих двух интервалах полей намагниченности слоев направлены противоположно друг другу), примерно в 2 раза больше наклона $d P / d H(H)$ в остальном диапазоне полей $(H>200$ Ое и $H<-230$ Ое $)$, в котором намагниченности обоих слоев сонаправлены.

Зависимость $d P / d H(H)$ для развертки магнитного поля слева направо имела такой же вид как и зависимость в случае развертки справа налево, и содержала три перехода в полях $-200,+25$ и +230 Ое. Вместе две зависимости $d P / d H(H)$ для прямой и обратной разверток магнитного поля образуют составную петлю гистерезиса (рис. 3,c), которая аналогично петле гистерезиса магнитного момента (рис. 3,d) содержит одну центральную петлю с центром в нулевом поле и коэрцитивной силой $\sim 25 \mathrm{Oе} \mathrm{и} \mathrm{две} \mathrm{боковых} \mathrm{петли} \mathrm{с}$ центрами в полях \pm 215 Ое и коэрцитивными силами $\sim 15$ Oe.

Таким образом, в экспериментах были получены зависимости производной отрицательного магнитосопротивления от магнитного поля, которые претерпевают скачки в критических полях, „опрокидывания“ намагниченности слоев, а также отображают магнитный гистерезис слоев. Образец, будучи частью микроволнового контура спектрометра, изменял электрическое сопротивление при изменении магнитного поля спектрометра, что влияло на добротность резонатора и записываемый сигнал первой производной микроволнового сопротивления образца.

\section{4. Обсуждение результатов}

Одним из наиболее известных механизмов изменения сопротивления проводящей среды в магнитном поле является положительное магнитосопротивление Лоренца, обусловленное сокращением траекторий движения электронов в магнитном поле [11]. Этот случай не реализуется в наших экспериментах, потому что при этом должна была бы наблюдаться возрастающая зависимость $d P / d H(H)$. Из рис. 3, $a, c$ следует, что в наших экспериментах эта зависимость убывающая и притом линейная. Этот случай отвечает отрицательному магнитосопротивлению, причем зависящему от поля квадратично: $\rho(H) \sim-H^{2}$. Можно предположить несколько разных механизмов магнитосопротивления, приводящих к такой зависимости. В [12] описан механизм отрицательного магнитосопротивления $\rho(H) \sim-H^{2}$, который учитывает сложение амплитуд вероятностей электронов по траекториям и изменение доли замкнутых орбит в магнитном поле, что свойственно системам низкой размерности. В [13-15] обсуждаются разнообразные варианты полевой зависимости отрицательного магнитосопротивления, обусловленного рассеянием носителей заряда на магнитных центрах (атомах примеси, ферромагнитных кластерах и т.п.). В рамках последнего механизма также встречаются зависимости $\rho(H) \sim-H^{2}$. Наконец, анизотропное магнитосопротивление зачастую также характеризуется квадратичной зависимостью от магнитного поля $[13,14]$. Не вдаваясь в подробности выбора между разными возможными механизмами магнитосопротивления (мы склоняемся к первому из перечисленных вариантов), сначала предложим объяснение полученного нами гистерезиса $d P / d H(H)$.

Рассмотрим сначала отдельный ферромагнитный слой (монослой) в магнитном поле. Индукция магнитного поля внутри каждого слоя $B_{01}=H+4 \pi M$ определяется суммой напряженности внешнего магнитного поля $H$ и намагниченности слоя $4 \pi M$. При увеличении внешнего магнитного поля, направленного против намагниченности слоя, происходит „опрокидывание“ магнитного момента, которое вызывает изменение индукции магнитного поля в слое $B_{02}=H-4 \pi M$. Магнитосопротивление определяется магнитным полем в слое и может быть записано в виде $\rho(B) \sim-B^{2}$. Поэтому изменение $B$ при перемагничивании слоя приводит к скачку сопротивления $\Delta \rho(B) \sim M H$. Производная сопротивления также претерпевает при этом скачок: $\Delta(d \rho(B) / d B) \sim M$. Экспериментальным доказательством этого факта для монослоя является рис. $3, a$, на котором наблюдается гистерезис величины $d P / d H$, который означает, что микроволновое сопротивление изменяется не только вследствие вариаций внешнего поля, но и вследствие изменения намагниченности образца.

Перейдем теперь к рассмотрению двойного ферромагнитного слоя (бислоя). По аналогии с монослоем в этом случае сопротивление каждого слоя по отдельности пропорционально квадрату индукции магнитного поля внутри слоя: $\rho_{1}(B) \sim-B_{1}^{2}, \rho_{2}(B) \sim-B_{2}^{2}$. Здесь и далее индекс 1 соответствует нижнему слою, индекс 2 - верхнему слою. Влиянием полей рассеяния, индуцируемых одним слоем в области другого слоя, можно пренебречь, так как данный эффект имеет место только на краях пластин и для тонких слоев (отношение толщины к латеральным размерам в нашем случае $\sim 10^{-6}$ ). В зависимости от взаимной ориентации намагниченностей двух слоев (имеется всего четыре возможности, см. рис. 1) возможны четыре случая поведения величины $d P / d H$

$$
\begin{aligned}
& (d P / d H)_{\uparrow \uparrow} \sim-2 H+4 \pi\left(M_{1}+M_{2}\right), \\
& (d P / d H)_{\uparrow \downarrow} \sim-2 H+4 \pi\left(M_{1}-M_{2}\right), \\
& (d P / d H)_{\downarrow \uparrow} \sim-2 H-4 \pi\left(M_{1}-M_{2}\right), \\
& (d P / d H)_{\downarrow \downarrow} \sim-2 H-4 \pi\left(M_{1}+M_{2}\right) .
\end{aligned}
$$

Направления стрелок в индексах $(d P / d H)$ соответствуют ориентациям намагниченностей слоев бислоя относительно положительного направления магнитного поля $H$. Левая стрелка соответствует намагниченности нижнего слоя, правая стрелка - намагниченности верхнего слоя. Изменения величины $d P / d H$ на переходах 
между состояниями бислоя определяются следующими соотношениями:

$$
\begin{gathered}
\Delta(d P / d H)_{\uparrow \uparrow \leftrightarrow \uparrow \downarrow}=\Delta(d P / d H)_{\downarrow \uparrow \leftrightarrow \downarrow \downarrow} \sim 4 \pi M_{2}, \\
\Delta(d P / d H)_{\uparrow \downarrow \leftrightarrow \downarrow \uparrow} \sim 4 \pi\left(M_{1}+M_{2}\right) .
\end{gathered}
$$

Согласно данным соотношениям, максимальный перепад производной поглощения микроволнового поля должен достигаться при одновременном перемагничивании обоих слоев (см. расчетную зависимость $d P / d H(\mathrm{H})$, кривая 1 на рис. $3, c)$, что и наблюдается в эксперименте. Таким образом, наличие центральных и боковых петель гистерезиса на зависимостях $d P / d H(H)$ и соотношение амплитуд этих петель объясняются скачкообразным изменением величины отрицательного магнитосопротивления бислоя при переходах между его магнитными состояниями.

В то же время наклон $d P / d H$ в состояниях $\uparrow \downarrow$ и $\downarrow \uparrow$ с антипараллельной ориентацией намагниченностей слоев отличается от наклона $d P / d H$ в состояниях $\uparrow \uparrow$ и $\downarrow \downarrow$, в которых намагниченности слоев сонаправлены. Это означает, что наряду с отрицательным магнитосопротивлением в исследуемых бислоях проявляется также положительный эффект ГМС, связанный с наличием спин-зависимого рассеяния носителей заряда в бислое. С учетом эффекта ГМС выражения (1) могут быть переписаны в виде

$$
\begin{gathered}
(d P / d H)_{\uparrow \uparrow} \sim-2 H+4 \pi\left(M_{1}+M_{2}\right), \\
(d P / d H)_{\uparrow \downarrow} \sim \alpha\left(-2 H+4 \pi\left(M_{1}-M_{2}\right)\right), \\
(d P / d H)_{\downarrow \uparrow} \sim \alpha\left(-2 H-4 \pi\left(M_{1}-M_{2}\right)\right), \\
(d P / d H)_{\downarrow \downarrow} \sim-2 H-4 \pi\left(M_{1}+M_{2}\right) .
\end{gathered}
$$

где $\alpha-$ коэффициент ГМС. В нашем случае $(d P / d H)_{\uparrow \downarrow} \sim 2(d P / d H)_{\uparrow \uparrow}$ (см. расчетную зависимость $d P / d H(H)$, кривая 2 на рис.3, $c)$, т. е. величина положительного ГМС бислоя составляет $\sim 100 \%$.

Таким образом, анализ зависимостей первой производной поглощения микроволнового поля $d P / d H$ от постоянного поля $H$ позволяет разделить вклады отрицательного магнитосопротивления отдельных слоев и положительного гигантского магнитосопротивления бислоя в целом. Амплитуда перепадов на зависимости $d P / d H(H)$ при перемагничивании слоев и наклон $d P / d H(H) \uparrow \uparrow$ в состоянии с параллельной взаимной ориентацией намагниченностей слоев определяются величиной их отрицательного магнитосопротивления, а соотношение наклонов $(d P / d H)_{\uparrow \downarrow}$ и $(d P / d H)_{\uparrow \uparrow}$ при двух различных взаимных ориентациях намагниченностей слоев - величиной положительного ГМС.

\section{5. Заключение}

Обнаружено, что изменение намагниченности ферромагнитных слоев гетероструктуры $\mathrm{MgO} / \mathrm{CoFeB} / \mathrm{Ta} /$ $\mathrm{CoFeB} / \mathrm{MgO} / \mathrm{Ta}$ с гигантским магнитосопротивлением приводит к соответствующему отклику добротности резонатора и микроволнового сопротивления образца, являющегося частью цепи микроволнового контура спектрометра ЭПР. Это позволяет с высокой точностью записывать петли магнитного гистерезиса образцов в спектрометре ЭПР и наблюдать скачкообразные изменения добротности контура при „опрокидывании“ намагниченности слоев в нарастающем (убывающем) магнитном поле спектрометра. На основе полученных результатов предложена альтернативная методика определения критических полей переключения магнитных состояний спиновых вентилей по изменению величины микроволнового поглощения в контуре, частью которого является образец. Полученные результаты открывают новые возможности для бесконтактного определения как сопротивления спиновых вентилей, так и их магнитного и соответствующего логического состояния.

\section{Список литературы}

[1] S. Ikeda, J. Hayakawa, Y. Ashizawa, Y.M. Lee, K. Miura, H. Hasegawa, M. Tsunoda, F. Matsukura, H. Ohno. Appl. Phys. Lett. 93, 082508 (2008).

[2] T. Zhu, P. Chen, Q.H. Zhang, R.C. Yu, B.G. Liu. Appl. Phys. Lett. 104, 202404 (2014).

[3] R. Soucaille, M. Belmeguenai, J. Torrejon, J.-V. Kim, T. Devolder, Y. Roussigné, S.-M. Chérif, A. A. Stashkevich, M. Hayashi, J.-P. Adam. Phys. Rev. B 94, 104431 (2016).

[4] S.D. Bader, Rev. Mod. Phys. 78, 1 (2006).

[5] D.B. Gopman, D. Bedau, S. Mangin, E.E. Fullerton, J.A. Katine, A.D. Kent. J. Appl. Phys. 115, 113910 (2014).

[6] S. Mangin, A. Sulpice, G. Marchal, C. Bellouard, W. Wernsdorfer, B. Barbara. Phys. Rev. B 60, 1204 (1999).

[7] H. Yang, M. Chshiev, B. Dieny, J. Lee, A. Manchon, K. Shin. Phys. Rev. B 84, 054401 (2011).

[8] C.-W. Cheng, C.H. Shiue, T.-I. Cheng, G. Chern. J. Appl. Phys. 112, 033917 (2012).

[9] A.I. Veinger, A.G. Zabrodskii, T.V. Tisnek. Phys. Status Solidi B 230, 107 (2002).

[10] R. Morgunov, M. Farle, M. Passacantando, L. Ottaviano, O. Kazakova. Phys. Rev. B 78, 045206 (2008).

[11] A.I. Veinger, A.G. Zabrodskii, T.V. Tisnek, S.I. Goloshchapov. Solid State Commun. 133, 455 (2005).

[12] А.И. Вейнгер, Т.В. Тиснек, И.В. Кочман, В.И. Окулов. ФТП 51, 172 (2017).

[13] К.П. Белов. УФН 164, 603 (2017).

[14] C. Butschkow, E. Reiger, A.Rudolph, S. Geißler, D. Neumaier, M. Soda, D. Schuh, G. Woltersdorf, W. Wegscheider, D. Weiss. Phys. Rev. B 87, 245303 (2013).

[15] А.И. Вейнгер, А.Г. Забродский, Т.В. Тиснек. ФТП 34, 774 (2000). 